\title{
159. Chromosomal Changes in Methylcholanthrene-induced Fibrosarcomas of the Mouse
}

\author{
By Yasuhiko KANEKo and Motomichi SASAKI \\ Chromosome Research Unit, Faculty of Science, Hokkaido University, Sapporo
}

(Comm. by Sajiro Makino, M. J. A., Dec. 12, 1975)

On the basis of karyotype analyses in some rat and Chinese hamster tumors induced by 7,12-dimethylbenz $(\alpha)$ anthracene (DMBA) or by Rous sarcoma virus, certain nonrandom chromosome changes occurring in them were postulated as specific for the oncogenic agents rather than the pathologic types of tumors (Mitelman et al. 1972). This hypothesis was further developed and applied to human neoplasms, although no specific etiologic agents have been identified in them (Rowley 1974). Referring to the obvious genetic heterogeneity of man, further systematic investigations with inbred animals are needed to generalize the concept of the agent-specific karyotypic changes.

We studied chromosomal changes in 14 methylcholanthreneinduced mouse sarcomas, by means of the quinacrine banding method.

Materials and methods. Three-month-old male mice of Swiss albino (SWJ) strain weighing 30-35 g were subcutaneously injected with $1 \mathrm{mg}$ of 20-methyl-cholanthrene (MC) dissolved in $0.1 \mathrm{ml}$ of olive oil. Fifteen tumors, which developed to $1.5 \mathrm{~cm}$ in diameter after 95 to 210 days of the MC injection, were excised and used. All tumors were histologically diagnosed as fibrosarcoma. All primary tumors and their cultures, except one culture line (MC11), were proven to be tumorigenic when transplanted into young SWJ mice.

Chromosomes were studied in direct fixations of the primary tumors as well as in their primary cultures within 12 days of in vitro propagation. The cultures were grown in Eagle's MEM supplemented with $15 \%$ fetal calf serum. The solid tumors were minced with scissors, incubated for 2 hours at $37^{\circ} \mathrm{C}$ in the culture medium containing colchicine, suspended in hypotonic $\mathrm{KCl}(0.075 \mathrm{M}$ for 20 minutes at $37^{\circ} \mathrm{C}$ ), fixed in $3: 1$ methanol-acetic acid, and then airdried on slides. The slides were stained with Giemsa or quinacrine mustard (Caspersson et al. 1970). The cultured cells were processed with essentially the same methods as above. Karyotypes were arranged by the proposed standard of Nesbitt and Francke (1973).

Results. Out of the 15 tumors, the chromosome numbers were countable in 14, while the remaining 1 (MC1) allowed no accurate 
counting on account of the extremely high proportion of cells with chromosome breakages. Most tumors were cytogenetically bimodal: 9 tumors showed their main mode in the diploid range, leaving 5 tumors in the tetraploid range. There was a tendency that cultured cells showed a wider distribution of chromosome numbers with an increased frequency of polyploid cells, as compared to direct preparations of primary tumors. This was most clearly shown in MC9; diploid cells occupied $100 \%$ of the direct sample, whereas they were reduced to $43 \%$ after 7 days of cultivation. The modal value of MC8 shifted from 40 to 41 after 5 days of cultivation. Details will be published elsewhere (Kaneko and Sasaki, in press).

A total of 63 metaphase plates were analyzed from 13 tumors (MC2-14), on the basis of Q-banding patterns (Table I). It was apparent that most tumors were karyotypically highly variable, even though there were some common karyotypic features within each tumor. As shown in Fig. 1, 23 marker chromosomes were found in

Table I. The results of karyotype analyses in 63 banded metaphases from $13 \mathrm{MC}$ tumors

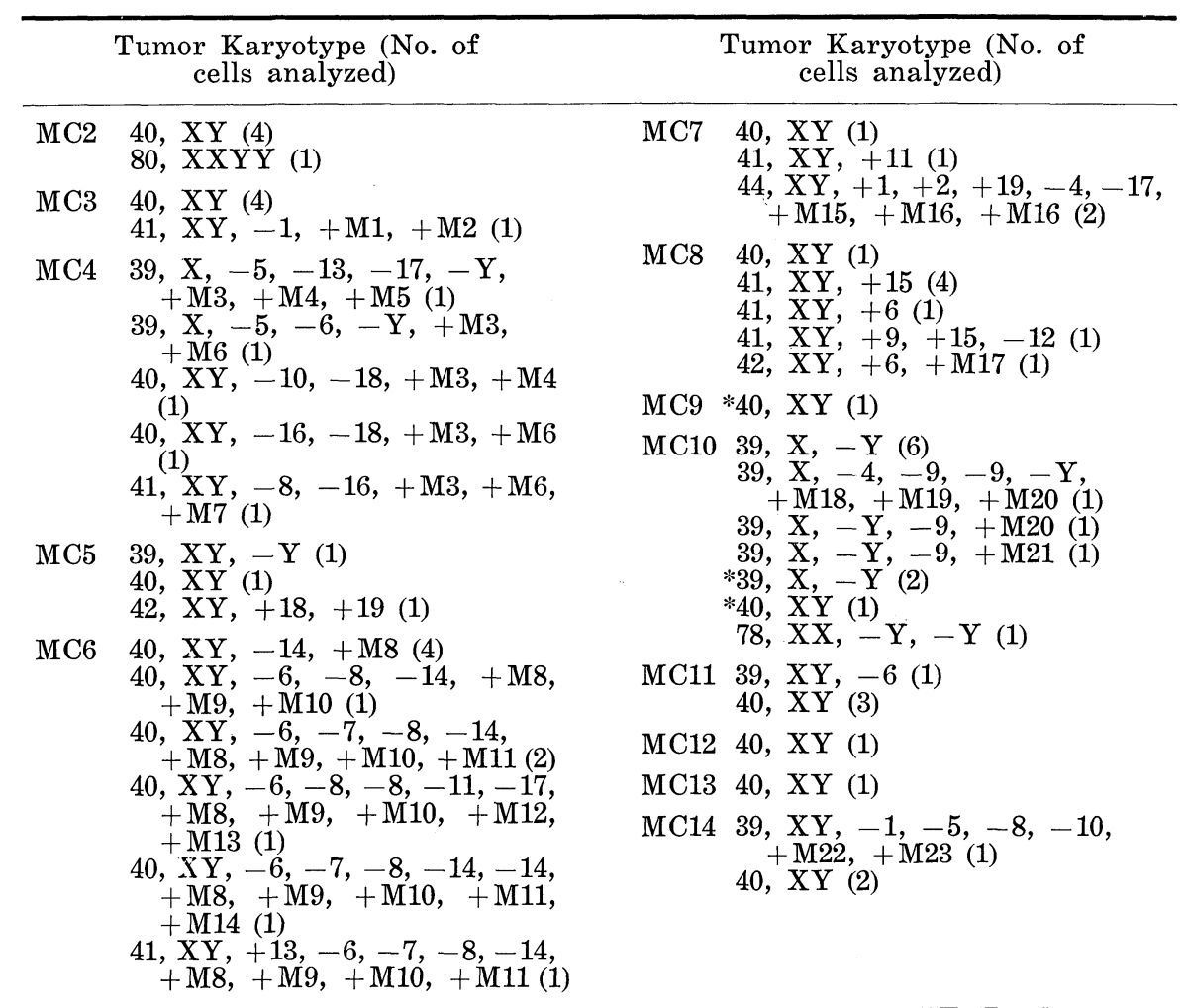

* Karyotypes were analyzed from direct preparations of primary tumors, all others being from cultured cells. 
7 tumors (MC3, 4, 6, 7, 8, 10 and 14), of which 16 were traceable, either totally or partially, by their characteristic banding patterns, while the remaining 7 were of unknown origin. Markers were different in different tumors. The markers of known origin were often associated with the loss or gain of certain normal elements which participated in the rearrangements. No markers were detected in 6 tumors, of which 4 (MC2, 9, 12 and 13) were karyotypically normal, so far as the present limited analyses were concerned. One of them (MC2) was assumed to have a tetraploid stemline. The remaining 2 tumors (MC5 and 11) showed only numerical variations.

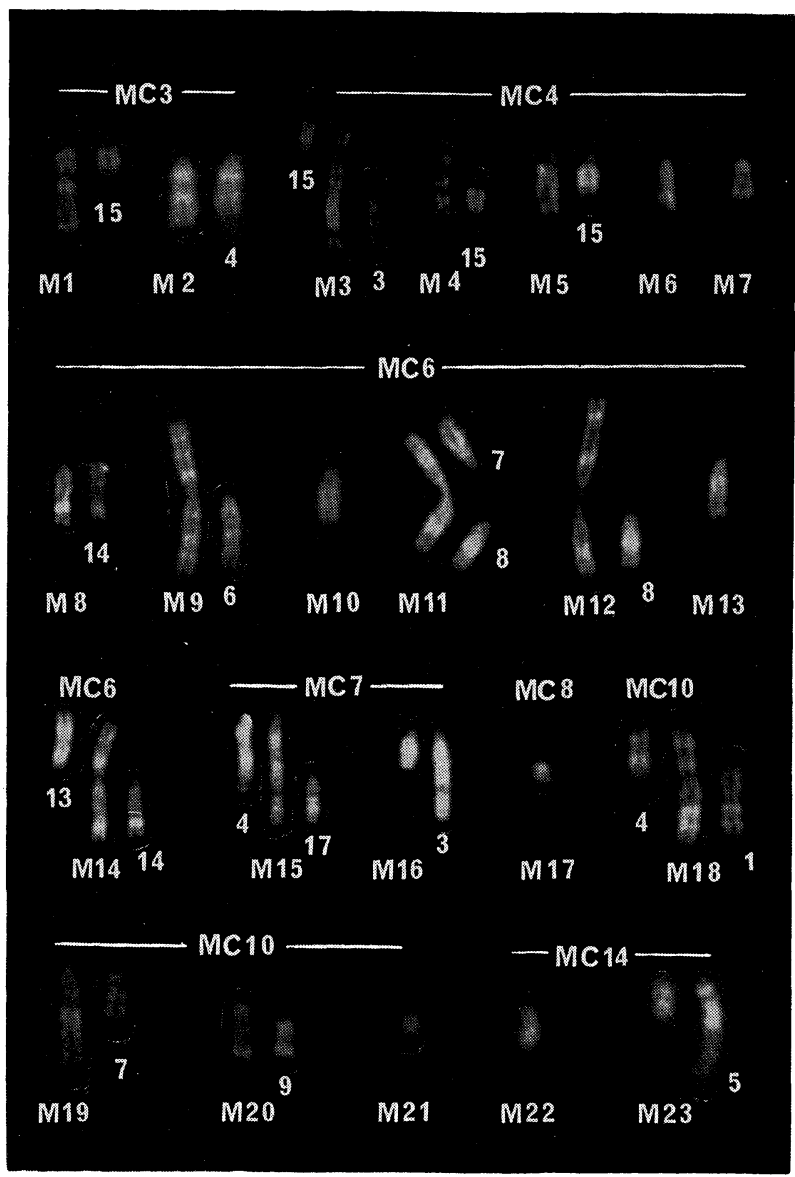

Fig. 1. Q-banding analyses of 23 marker chromosomes (M1-23) from the $7 \mathrm{MC}$ tumors under study. For explanation of their derivation, the corresponding normal copies are shown together from the same metaphase plates.

Remarks. We failed to detect any specific karyotypic change shown by non-random clustering of additional or missing chromo- 
somes, or chromosomal segments, in the MC-induced mouse sarcomas here studied, taking both numerical and structural variations into account. This seems to be not consistent with the results obtained in rat sarcomas induced by MC and benzpyrene (BP), where certain nonrandom variations occurred (Levan and Levan 1975). However, the specific chromosomal changes in the $\mathrm{MC}$ - and $\mathrm{BP}$-induced rat sarcomas were less conspicuous as compared to those seen in DMBAinduced leukemias (Kurita et al. 1968) and sarcomas (Mitelman et al. 1972) of the rat. Levan and Levan (1975) suggested that this fact was associated with the differences in oncogenic capacity among the 3 compounds. On the other hand, Olinici and DiPaolo (1974) failed to confirm the results of Mitelman et al. (1972) in rat sarcomas induced by both in vivo and in vitro applications of DMBA. Hellström (1959) studied, without banding analyses, 14 MC-induced primary fibrosarcomas of mice, and reported that most of them were characterized by aneuploid stemlines with a considerable variability in chromosome number. We confirmed this and presented further evidence of the cytogenetic heterogeneity which was more clearly demonstrated by banding analyses. It should be mentioned, however, that our banding analyses were made mostly on cultured cells in which the chromosome-number variation was more or less widened, even in very early stages of primary cultures. While the relevance of cultured cells for evaluating the chromosome constitution of solid tumors has been suggested by Levan (1974) following a study with $\mathrm{BP}$-induced rat tumors, the situation does not seem to be the same in the mouse. Thus, many problems remain to be solved in the future.

We are grateful to Emeritus Prof. S. Makino, Hokkaido Univ., and to Dr. M. Hosokawa, Cancer Inst., Hokkaido Univ. School of Med., for pathological diagnosis of the tumors. Contributions from the Chromosome Research Unit, Hokkaido Univ., supported by grants from the Ministry of Education, Science, and Culture, Japan.

\section{References}

Caspersson, T., Zech. L., Johansson, C., and Modest, E. J. (1970) : Chromosoma (Berl.), 30, 215-227.

Helström, K. E. (1959) : J. Nat. Cancer Inst., 23, 1019-1033.

Kaneko, Y., and Sasaki, M.: Chromosome Information Service, in press.

Kurita, Y., Sugiyama, T., and Nishizuka, Y. (1968) : Cancer Res., 28, 1738-1752. Levan, G. (1974) : Hereditas, 78, 273-290.

Levan, G., and Levan, A. (1975): Hereditas, 79, 161-198.

Mitelman, F., Mark, J., Levan, G., and Levan A. (1972) : Science, 176, 1340-1341. Nesbitt, M. N., and Francke, U. (1973) : Chromosoma (Berl.), 41, 145-158. Olinici, C. D., and DiPaolo, J. A. (1974) : J. Nat. Cancer Inst., 52, 1627-1634. Rowley, D. J. (1974) : J. Nat. Cancer Inst., 52, 315-320. 